CI:24.0-26.6); p<0.001], USA; 24\%(95\%CI:21.9-25.3) vs. $16 \%[(15.4-16.9) ; \mathrm{p}<0.001])$, and in males (Ireland; 8\%[(95\% CI:5.3-10.8) vs. $7 \%[(95 \% \mathrm{CI}: 6.1-7.9) ; \mathrm{p}=0.644]$, England; $30 \%(95 \% \mathrm{CI}: 25.8-33.9)$ vs. $17 \%(95 \% \mathrm{CI}: 15.7-18.3) ; \mathrm{p}<0.001]$, USA; $16 \%(95 \% \mathrm{CI}: 14.4-17.7)$ vs. $11 \%[(95 \% \mathrm{CI}: 10.6-12.2)$; $\mathrm{p}<0.001]$. After adjusting for all model variables, people with diabetes had 1.5 increased odds of depression compared to people without diabetes [(95\%CI:1.4-1.6), $\mathrm{p}<0.001]$.

Conclusion In older people in three countries with different health systems, depressive symptom prevalence was (1) consistently higher among men and women with diabetes than nondiabetes counterparts and (2) varied across health systems. While use of self-report data may limit the accuracy of the results, use of the CESD to categorise depression and of large nationally representative datasets strengthens the study. Interrogation of additional country-level factors associated with depression will further explain variation in depressive symptom prevalence across health systems.

\section{OP96 ARE WOMEN WITH CHRONIC POOR MENTAL HEALTH LESS LIKELY TO ATTEND BREAST SCREENING AND DOES THIS EXPLAIN THE SOCIAL AND GEOGRAPHIC VARIATIONS IN UPTAKE? A POPULATION-WIDE RECORD LINKAGE STUDY}

${ }^{1} \mathrm{D}$ O'Reilly*, ${ }^{1} \mathrm{E}$ Ross, ${ }^{1} \mathrm{~A}$ Maguire, ${ }^{1} \mathrm{M}$ Donnelly, ${ }^{2} \mathrm{~A}$ Mairs, ${ }^{2} \mathrm{C}$ Hall, ${ }^{1} \mathrm{D}$ O'Reilly. ${ }^{1} \mathrm{C}$ entre for Publich Health, Queen's University Belfast, Belfast, UK; ${ }^{2}$ Public Health Agency, Belfast, UK

\subsection{6/jech-2019-SSMabstracts.90}

Background Research from the United States (US) provides compelling evidence of disparities in breast screening uptake for women with mental illness, yet few attempts have been made to examine this association in the United Kingdom (U. $\mathrm{K})$ where healthcare is free at the point of use. It is well established that mental illness is not evenly distributed across the population. For example, mental illness is more prevalent in individuals who are unmarried, socially deprived, and residing in urban areas. Interestingly, these attributes are also strong predictors of lower attendance at breast screening, and it is possible that this may be explained by the increased prevalence of mental illness in these individuals. This study aims to examine the impact of self-reported poor mental health on attendance at breast screening in the United Kingdom (UK), and to what extent this explains socio-demographic inequalities in uptake.

Methods Breast screening records were linked to 2011 Census data within the Northern Ireland Longitudinal Study (NILS). This identified a cohort of 57,328 women who were followed through one complete three-year screening cycle of the National Health Service (NHS) breast screening programme. Poor mental health was identified using responses to question 23 of the Northern Ireland 2011 Census which asked, 'Do you have any of the following conditions, which have lasted, or are expected to last, at least 12 months?' to which 'An emotional, psychological or mental health condition (such as depression or schizophrenia)' was a possible response. Information on individual and household-level attributes was also derived from Census records. Logistic regression was employed to calculate odds ratios (ORs) and 95\% confidence intervals of attendance at breast screening.

Results $10.7 \%$ of women in the cohort reported poor mental health, and in fully-adjusted analyses, these individuals were
23\% less likely to attend breast screening (OR 0.77: $0.73-$ 0.82). Although poor mental health was a strong predictor of screening uptake, it only explained a minimal degree of the observed inequalities in uptake by socio-economic status and marital status, and did not explain any of the variation by area of residence. Furthermore, there was no evidence of effect modification between poor mental health and any other socio-demographic determinant of screening uptake.

Conclusion This study provides novel evidence of inequalities in breast screening uptake for women with self-reported poor mental health in the UK. Targeted interventions are required to improve screening uptake in individuals with mental illness to optimise the mortality benefits achieved through population-wide screening.

\section{Friday 6 September Tobacco Control}

\section{OP87 A COMPREHENSIVE EVALUATION OF THE IMPACT OF RECENT ENGLISH TOBACCO CONTROL POLICY USING SECONDARY DATA}

${ }^{1} \mathrm{~K}$ Eminson*, ${ }^{2} \mathrm{D}$ Gillespie, ${ }^{1} \mathrm{~S}$ Lewis, ${ }^{1} \mathrm{M}$ Opazo-Breton, ${ }^{1} \mathrm{I}$ Bogdanovica, ${ }^{2} \mathrm{~A}$ Brennan, ${ }^{1} \mathrm{~J}$ Britton, ${ }^{3} \mathrm{Q}$ Wu, ${ }^{2} \mathrm{P}$ Meier, ${ }^{2} \mathrm{~J}$ Holmes, ${ }^{1} \mathrm{~T}$ Langley. ${ }^{1}$ Division of Epidemiology and Public Health, University of Nottingham, Nottingham, UK; ${ }^{2}$ School of Health and Related Research, University of Sheffield, Sheffield, UK; ${ }^{3}$ Department of Health Sciences, University of York, York, UK

\subsection{6/jech-2019-SSMabstracts.91}

Background Smoking is the biggest avoidable cause of death and disability in England. A range of laws and policies aimed at preventing this harm have been introduced in England to try to prevent young people from becoming smokers, and encourage existing smokers to quit and to protect others from the harmful effects of cigarette smoke. This Study aimed to evaluate the effects of these policies using publically available data.

Methods We developed logic models for each policy that indicated the anticipated causal pathways for each policy and used these to develop hypotheses for our analysis. Interrupted time series analysis was carried out systematically and using a consistent approach across policies, datasets, outcomes and populations. Outcome measures were adult smoking prevalence, quitting behaviour and consumption. Models were adjusted for sociodemographic factors, e-cigarette prevalence and mass media expenditure. Datasets included the Smoking Toolkit Study (STS) and the Health Survey for England (HSE).

Results Following a point of sale display ban in large shops in April 2012, based on the STS data, there was a significantly steeper declining trend in adult smoking prevalence. This finding was supported by results from the HSE. A similar result was found when analysing quit attempts. Following a point of sale ban in small shops in April 2015, there was a significantly steeper decline in trend in adult prevalence. There was also a significant decline in trend in quit attempts. No significant impact of the smoke-free policy on smoking prevalence was found and we found no evidence of a combined impact of three policies that were implemented in October 2015 (proxy purchase ban, minimum age of purchase for e-cigarettes and smoking ban in cars carrying children) on adult prevalence. 